\title{
Impact of a 3-weeks randomized double-blind cross-over study curuminoid supplementation on endotoxemia, inflammatory markers, and lipid profiles in healthy overweight and obese adults
}

\author{
M. Nuraiza, C.A. Edwards and E. Combet \\ Human Nutrition, School of Medicine, College of MVLS, University of Glasgow, Glasgow G31 2ER, UK.
}

Postprandial endotoxaemia (increased bacterial lipopolysaccharide [LPS] level in the circulation) is associated to the increase of pro-inflammatory markers after intake of high-fat high-calorie meals ${ }^{(1)}$. Endotoxaemia is a potential driver for chronic low-grade inflammation, linked to non-communicable chronic diseases. Curcumin (turmeric) has potential anti-inflammatory and hypolipidemic properties; and was shown to attenuate the effect of LPS-induced endotoxaemia in rats ${ }^{(2)}$. The aim of this study was to investigate whether curcuminoid supplementation affected postprandial endotoxaemia, inflammatory markers, and lipid profiles in humans.

Healthy volunteers $\left(\mathrm{n}=16,50 \%\right.$ men and $50 \%$ women, aged $19-43 \mathrm{y}$, BMI $25-44 \mathrm{~kg} / \mathrm{m}^{2}$, fat mass $\left.19-53 \%\right)$ participated in a double-blinded, placebo-controlled, cross-over study (4 weeks wash-out period). Participants were randomized to 1 capsule per day, curcuminoids $(380 \mathrm{mg}$ ) or placebo, for three weeks. Postprandial endotoxaemia was induced by single high-fat high-calorie meal intake (929 kcal, $65 \mathrm{~g}$ fat, $63 \% \mathrm{E}$ ). Blood samples were collected before and after each leg of the study. Endotoxaemia markers (sCD14 \& LBP) and inflammatory markers (CRP, TNF- $\alpha$, IL-6, IL-1 $\beta$, and IL-10) were measured with immunoassays; lipids were measured colorimetrically.

Two participants dropped-out. There was no change in LBP for either trial leg. Subgroup analysis (3) however indicated a $22 \%$ decrease in LBP in volunteers with very high fat mass $(n=5)$ after leg A $(p=0.02)$. No differences were seen in CRP level after either leg, with large inter-individual variability $(36 \cdot 4-1028 \cdot 3 \mathrm{ng} / \mathrm{mL})$. sCD14 decreased after both legs $($ leg $A, p=0.015 ;$ leg $B$, $p=0 \cdot 019)$. There were no effects on TNF- $\alpha$, IL-6, and IL-1 $\beta$. HDL level (before high-fat meal) was significantly higher after leg A ( $p=0 \cdot 01$ ) with no different after the meal. No other effects were seen on total cholesterol, LDL, and triglycerides.

Table 1. Changes in CRP, LBP, and sCD14 levels $(\Delta)$ after leg A and B. *significant differences within leg (paired t-test), p values are differences between legs (independent t-test)

\begin{tabular}{|c|c|c|c|c|c|c|c|c|}
\hline \multirow[b]{2}{*}{ Leg } & \multirow{2}{*}{$\begin{array}{l}\text { Assay } \\
\text { Time point (hr) }\end{array}$} & \multirow{2}{*}{$\begin{array}{c}\Delta \mathrm{CRP}(\mathrm{ng} / \mathrm{mL}) \\
0\end{array}$} & \multicolumn{2}{|c|}{$\Delta \mathrm{LBP}(\mu \mathrm{g} / \mathrm{mL})$} & \multicolumn{2}{|c|}{$\Delta \mathrm{sCD} 14(\mathrm{ng} / \mathrm{mL})$} & \multicolumn{2}{|c|}{$\Delta \mathrm{HDL}(\mathrm{mg} / \mathrm{dL})$} \\
\hline & & & 0 & 3 & 0 & 3 & 0 & 3 \\
\hline $\mathbf{A}$ & Mean & -58.63 & -0.41 & -0.07 & $-136 \cdot 10^{*}$ & -45.02 & 3.99 & 2.46 \\
\hline & $\mathrm{SD}$ & $316 \cdot 15$ & 1.04 & 0.55 & 189.08 & 185.56 & $7 \cdot 38$ & 5.48 \\
\hline B & Mean & $110 \cdot 66$ & 0.04 & -0.08 & $-125 \cdot 34^{*}$ & $-52 \cdot 41$ & $-3 \cdot 4^{*}$ & $-1 \cdot 26$ \\
\hline & $\mathrm{SD}$ & $249 \cdot 53$ & $0 \cdot 43$ & 1.29 & 183.63 & 232.54 & $5 \cdot 85$ & $6 \cdot 65$ \\
\hline & $\mathrm{p}=$ & $0 \cdot 16$ & 0.22 & 0.99 & 0.87 & 0.92 & 0.01 & $0 \cdot 12$ \\
\hline
\end{tabular}

HDL is the main lipoprotein removing LPS from the circulation, transporting LPS to the hepatocytes for clearance (4). Thus increased HDL level could be of benefit to reduce LPS and inhibit subsequent inflammatory responses. Assessing LPS level in plasma will give a better understanding of this effect.

1. Laugerette F, Vors C, Géloën A et al. (2011) J Nutr Biochem 22, 53-59.

2. Chen HW, Kuo HT, Chai CY et al. (2007) J Endotoxin Res. 13(1), 15-23.

3. Gallagher D, Heymsfield SB, Heo M et al. (2000) Am J of Clin Nutr 72(3), 694-701.

4. Manco M, Putignani L, and Bottazzo GF (2010) Endocrine Rev 31(6), 817-844. 